\title{
An endoscopic technique for treating symptomatic distal jejunum obstruction by leaving the overtube in place
}

\author{
Enrique Pérez-Cuadrado ${ }^{1}$, Fernando Carballo ${ }^{2}$, Rafael Latorre ${ }^{3}$, Federico Soria ${ }^{4}$ \\ and Octavio López-Albors ${ }^{3}$
}

${ }^{1}$ Unit of Endoscopy. Hospital Morales Meseguer. Murcia, Spain. ${ }^{2}$ Department of Gastroenterology. Hospital Virgen de la Arrixca. Murcia, Spain. ${ }^{3}$ Department of Anatomy. Shool of Veterinary. Murcia, Spain. ${ }^{4}$ Unit of Endoscopy. CCMI.

Cáceres, Spain

\begin{abstract}
Double balloon enteroscopy has a limitation for positioning an enteral stent in the distal jejunum through its long, narrow biopsy channel. When the distal end of its overtube is left in place close to the neoplasia with the enteroscope removed, if we push an enteral stent introduction system, it tends to form loops so the technique cannot be performed with this instrument. However, the double balloon colonoscope has a shorter overtube length and using the same push-and-pull technique we can reach the distal jejunum with this instrument by inserting the delivery stent system without loops through its overtube. We present a patient with neoplastic obstruction in the distal jejunum with resolution of his symptoms after positioning an enteral stent.
\end{abstract}

Key words: Double ballon enteroscopy. Distal jejunum. Neoplastic obstruction.

\section{INTRODUCTION}

Distal jejunum obstruction (DJO) is a rare but severe complication of small bowel (SB) adenocarcinoma. Surgery is the gold standard if palliative treatment is needed, because with the use of conventional oral endoscopy or push enteroscopy it is difficult to reach the deep SB. Double balloon enteroscopy (DBE) has recently become the new first line diagnosis and treatment in SB (1) with the use of a long

Pérez-Cuadrado E, Carballo F, Latorre R, Soria F, López-Albors $O$. An endoscopic technique for treating symptomatic distal jejunum obstruction by leaving the overtube in place. Rev Esp Enferm Dig 2013;105:107-109.

Received: 10-04-2012

Accepted: 23-08-2012

Correspondence: Enrique Pérez-Cuadrado. Unit of Endoscopy. Hospital Morales Meseguer. Avda. Marqués de los Vélez, s/n. 30008 Murcia, Spain e-mail: eperezcuadradom@gmail.com enteroscope through an overtube. There are though, technical limitations if a stent is placed due to the long, narrow biopsy channel $(2.8 \mathrm{~mm}$ and 2,000 $\mathrm{mm})$ compared with the short length and external thickness of available stent introduction systems (1,800 mm length). The DBE overtube measures $1,350 \mathrm{~mm}$, making it too long for this endoscopic therapy because it can make the introduction of a stent delivery system difficult due to the friction between both instruments and the formation of loops while pushing.

A new instrument for DBE is the double balloon colonoscope (DBC), which uses the same push-and-pull technique, but has a shorter overtube $(950 \mathrm{~mm})$ and endoscope $(1,520 \mathrm{~mm})$ with the same biopsy channel as the DBE. We present a case of DJO successfully palliated with the use of DBC.

\section{CASE REPORT}

A 42-year-old male patient with liver metastasis due to adenocarcinoma was referred for DJO. He was suffering uncontrollable vomiting and abdominal pain. An abdominal CT scan revealed a distal jejunum stenosis and proximal SB fluid levels. Capsule endoscopy [Given imaging (R) Endotécnica. Madrid] was indicated to clarify the benign or malignant origin of this stenosis in order to decide upon the appropriate therapeutic management, and this showed that the origin of the primitive tumor was probably neoplastic. On finding this, oral DBC, using the classic push-andpull technique (EC-450B15. Fuji Film. ST Endoscopy. Spain) was indicated for palliation treatment. A nasogastric tube was inserted and the endoscope with its overtube (TS13101. Fuji Film. ST Endoscopy. Spain) was introduced orally. After a first endoscopic inspection of the gastric and duodenal lumen, there was no fluid retention so the nasogastric tube was removed and endotracheal intubation was not required. With the patient under deep sedation, the steno- 
sis was reached, $250 \mathrm{~cm}$ from the angle of Treitz, showing retention of the endoscopic capsule near a complete tumor obstruction (Fig. 1). Under radiological control, a sphincterotome introduced via the biopsy channel of the DBC directed a 0.35 inch wire guide that crossed the tumor reaching the distal SB. The sphincterotome was then introduced through the tumor via the guide wire, and allowed the passage of contrast, showing distal permeability of the SB and a $5 \mathrm{~cm}$ long stenosis. With radiological control, the capsule endoscopy (radiopaque) was close to the proximal stenosis but presented retrograde displacement with the endoscopic manipulation, so an external skin marker was positioned over the proximal site of the stenosis. After withdrawal and rectification of both instruments (overtube and enteroscope) with inflated balloons, the DBC was then removed while the inflated overtube balloon was kept close to the tumor under fluoroscopic control. In this maneuver the guide wire was simultaneously pushed to maintain its position distal to the stenosis. Then, a lubricated enteral stent delivery system (Taewoongmedical. Corea) $(12 \mathrm{~cm} \mathrm{x} 20 \mathrm{~mm}$ ) was advanced over the guidewire without difficulty through the straightened overtube left in place. The stent crossed the tumor, located distal to the external marker under X-ray control (Fig. 2). The deployment of the stent was easily controlled with this skin marker reference, and its proximal cup was placed above the stenosis. After removal of the introduction system and guidewire, the DBC was reintroduced through the overtube with an inflated balloon, confirming the correct placement and patency of the stent, and the contrast being freely passed to the ileum (Fig. 3). After this, biopsies were taken through the mesh. The biopsy was not performed before the stent had been placed in order to avoid the possibility of bleeding which would have made the passage of the guidewire through the tumor difficult.

Later, the endoscopy capsule and the distal cap of the DBC (that had been left as foreign bodies after endoscope withdrawal) were extracted with a Roth net. As the endoscopic capsule did not pass through the overtube, the

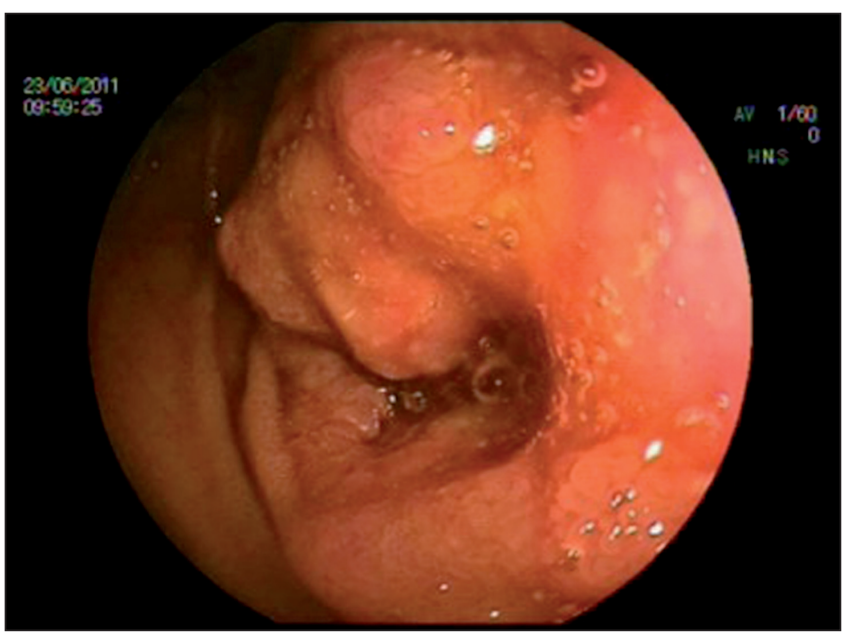

Fig. 1. Total obstruction by jejunal adenocarcinoma
DBC and overtube (with deflated balloon) were removed simultaneously.

The pathological study of the biopsies confirmed adenocarcinoma. The X-ray control showed no levels and a good stent deployment (Fig. 4) and the patient improved clinically and was discharged home the next day. The patient finally died a month later with good small bowel transit.

\section{DISCUSSION}

An alternative to surgery in the case of symptomatic malignant SB obstruction is nasogastric or preferably, long decompression tubes (such as the Miller Abbott tube), but often these do not pass into the SB and can sometimes form

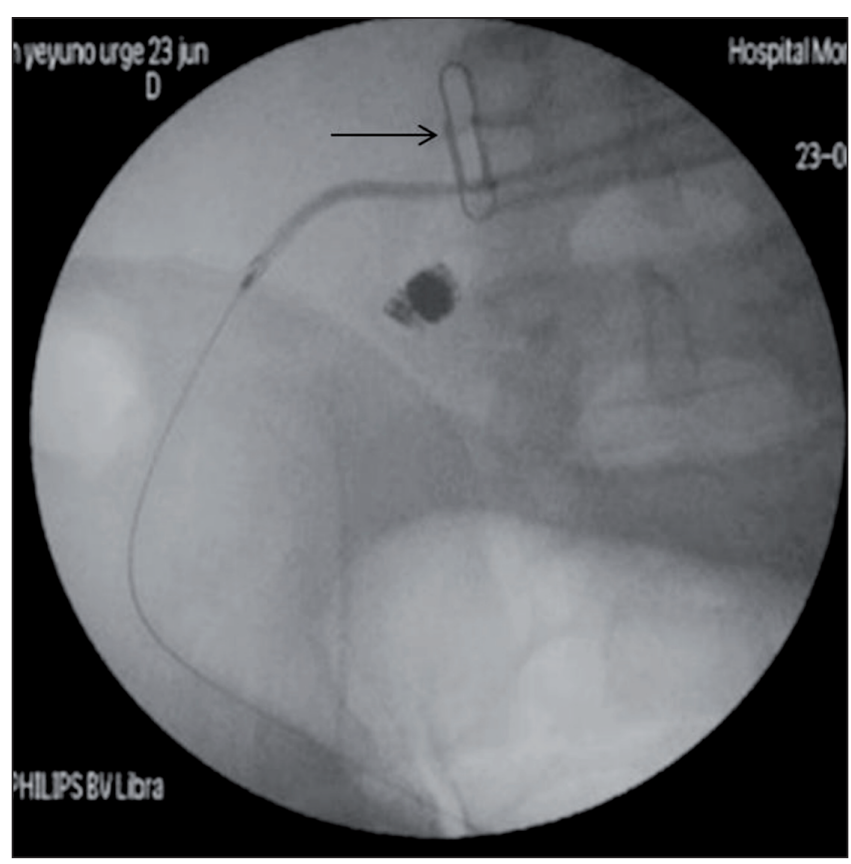

Fig. 2. Enteral stent system over the guide wire through the overtube (arrow, skin marker of the tumoral stenosis).

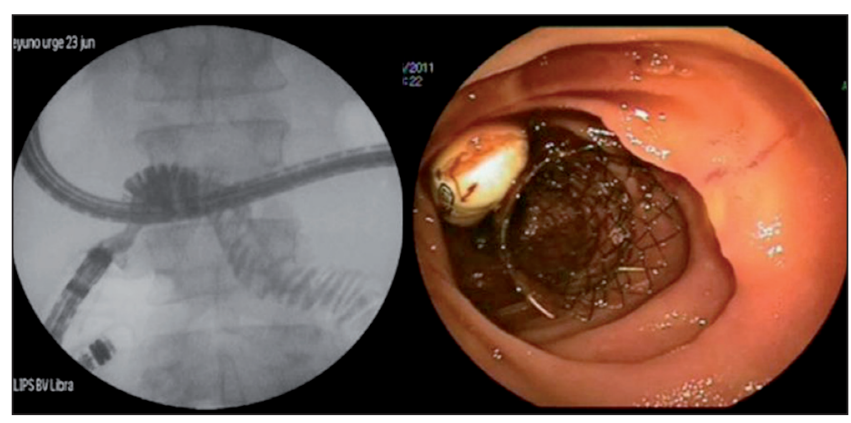

Fig. 3. DBC through enteral stent (arrows) with contrast instillation (left) and permeability of distal SB. The proximal part of the stent compresses the capsule, which could be withdrawn. 


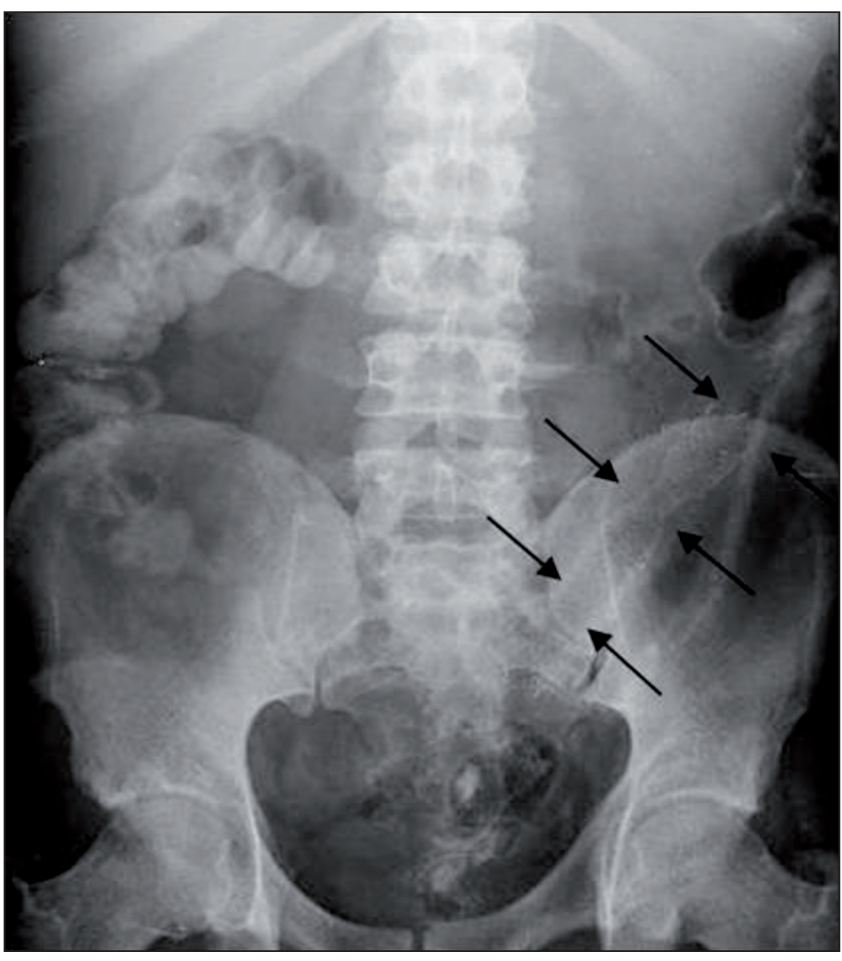

Fig. 4. Expanded stent (arrows) in distal jejunum the day after placement with resolution of the obstruction. Contrast media in the colon.

knots and be difficult to remove. Thus, special tubes are now rarely used, and only in selected cases. Upper endoscopy with a colonoscope, or push enteroscopy have limitations to reach deep into the SB. SB stent insertion for palliation in cases where there is to be no surgical management of the distal jejunal adenocarcinoma obstruction is an attractive idea but there are also technical limitations. In a systematic review of data over the first decade of use (2) SB obstruction represents $5.8 \%$ of the total indications in more than 12,000 DBE procedures. In a symptomatic patient with deep SB neoplastic findings, there are no specific dedicated TTS SB stents for DBE placement due to its long, narrow biopsy channel. On the other hand, we need balloon-assisted or spi- ral enteroscopy, to orally reach as far as the distal jejunum. Ross et al. (3) described the first stent placement in SB -using an esophageal stent- with DBE using this technique, but in a difficult case with long stenosis in the descending duodenum. SB stent placement with spiral enteroscopy has also been described by Lennon et al. (4), but at the level of the angle of Treitz and proximal jejunum (two cases) with good results. The spiral system has a theoretical advantage because it keeps its special overtube placed near the tumor, but this technique has lower insertion than DBE (5). In fact, there are no cases of cecal intubation orally in spiral enteroscopy, therefore, it seems that for deep insertion, the technique of choice at the moment is DBE. But the diameter of the DBE endoscope does not allow a delivery stent system to be passed, and its overtube $(1,350 \mathrm{~mm})$ is too long for this. This new instrument, the DBC has the advantage of having a shorter overtube $(950 \mathrm{~mm})$ that allows an enteral delivery system to be passed, and a shorter biopsy channel $(1,520$ $\mathrm{mm}$ ), that allows conventional accessories like sphincterotomes and the Roth net to be passed. The DBC, with the same push-and-pull technique is able to reach the whole of the jejunum. Using the fixed overtube with an insuflatted balloon, we can introduce available dedicated self-expandable enteral stents. In conclusion, DBC was useful to place a stent with enteral design in the deep SB.

\section{REFERENCES}

1. Pérez-Cuadrado E, Mas P, Hallal H, Shanabo J, Muñoz E, Ortega I, et al. Double balloon enteroscopy: a descriptive study of 50 explorations. Rev Esp Enferm Dig 2006;98:73-81.

2. Xin L, Liao Z, Jiang YP, Li ZS. Indications, detectability, positive findings, total enteroscopy, and complications of diagnostic double balloon enteroscopy: A systematic review of data over the first decade of use. Gastrointest Endosc 2011;74:563-70.

3. Ross A, Semrad C, Waxman I, Dye C. Enteral stent placement by double balloon enteroscopy for palliation of malignant small bowel obstruction. Gastrointest Endosc 2006;64:835-6.

4. Lennon AM, Chandrasekhara V, Shin EJ, Okolo PI 3rd. Spiralenteroscopy-assisted enteral stent placement for palliation of malignant small-bowel obstruction. Gastrointest Endosc 2010;71:422-5.

5. Pérez-Cuadrado E, Esteban P, Shanabo J. Enteroscopia de doble balón vía oral hasta ciego. Rev Esp Enferm Dig 2007;99:351. 